\title{
The research of Crop breeding technical architecture based on Cyber-Physical System(CPS)
}

\author{
Yue $\mathrm{E}^{1, \mathrm{a}}$, Ye Ping Zhu ${ }^{2, \mathrm{~b}}$ \\ ${ }^{1,2}$ Research Institute of Agriculture Information, Chinese Academy of Agricultural Science, Key \\ Laboratory of Agri-information Service Technology, Ministry of Agriculture, Beijing, China,100081.
}

aeyue@caas.cn, bzhuyeping@caas.cn Keywords: Crop breeding, CPS(Cyber-Physical System), Intellectualization, Informatization,
Collaboration services

\begin{abstract}
In China, under the background of improved about concentration degree of crop breeding enterprise and technology of crop breeding, it was the inevitable choice that carry out crop breeding technology innovation, strengthen information communication, joint crop breeding, and promoted the core competitiveness of crop breeding enterprise. For this purpose, first, this paper introduced the present situation of crop breeding management and technology application in China. And then, according to the characteristics of the CPS(Cyber-Physical System), the goal was intelligent and information of the whole process of crop breeding, research the conjunction point about CPS and crop breeding management. At last, put forward the intelligent and information model of crop breeding based on the CPS framework. The model framework consider the characteristics of personalization, integration and diversity in the whole process of crop breeding, both support collaborative control and cooperation between physical entity (such as field acquisition equipment, laboratory analysis equipment, etc.), and consider service about multi-layered decision based on crop breeding data mining. Through crop breeding based on the CPS system architecture, for the vast number of crop breeding enterprises, crop breeding research institutes, and so on, it could realize the resource sharing, collaborative breeding and joint test, thus promote the development of crop breeding in China, so the paper has the reference significance.
\end{abstract}

\section{Introduction}

Crop breeding was a heavy scientific research and production practice, the practice proved that breeding fine varieties was the fundamental way to realize increase production of grain, to ensure food security, increase agricultural output, eased the contradiction between population and land in our country. Therefore, the application of modern science and technology to develop crop breeding special equipment and data analysis system, both enhancing the technical levels of crop breeding, speed up the process of our modern agricultural science and technology, and improve the ability of the sustainable development of agriculture in our country.

For crop breeding, abroad crop breeding enterprise had begun to apply modern information technology, sensor technology, automation technology and so on[1-5], these technology applied in different processes of crop breeding, for example, many of photoelectric detection technology be used in crop quality and shape detection, and develop a series of plants comprehensive characteristic testing equipment in the field, and so on. At the same time, crop breeding experimental design and data management software was developed in the US, Canada, Netherlands and so on, and these physical equipment and management software to realize a large number of crop breeding data management and sharing, crop breeding information level was greatly increased.

Comparison of crop breeding enterprise in China[6-9], on the one hand, according to reports that have nearly hundred large crop breeding enterprise as of 2016, these large crop breeding enterprise had been basically set up commercialization crop breeding technological system, basically satisfy the needs of information and automation level crop breeding, but the crop breeding process, management pattern compared with foreign crop breeding technological, there was still a large gap. On the other hand, with the developing of crop breeding enterprise in China, the majority of 
enterprises and scientific research units would focusing on one or several links of the whole process in the crop breeding, and ultimately become some professional team through business transformation and technology upgrading, such as germplasm improvement, the joint test and molecular detection, etc. Thus, under the trend of specialization and socialization collaboration, must be strengthening union and complementary advantages, ultimately ability to form synergy and competitive advantage. In recent years, the CPS was not only caused the wide attention in the relevant government departments and scientific research institutions, but also has become a important study direction. As the evolution of the Internet of things, the CPS has better adaptability and collaborative, it could realize the optimal control between people and objects and communication, and protection system efficient real-time implementation of the related function, and provides more convenient and intelligent service to people. Therefore, it was important meaning that the research and application of crop breeding based on CPS.

\section{The present situation of crop breeding management technology in China}

With the rapid development of technology about wireless sensor network, Internet of things, embedded systems, automatic control systems etc, in the whole process of crop breeding, different scientific research units and relevant enterprises had purchase and establish various of physical entity devices, these devices had the ability of collection and analysis of different stages data for crop breeding. These equipment have differentiated communication ability, computation ability and cognitive ability, some of the field data collection devices, and some of the laboratory data analysis devices[10-13]. How to manage these instruments and equipment, how to aimed to specific crop breeding requirements to choose appropriate equipment, and how to effective complete crop breeding task was an important problem. Now, these physical entity dispersed in different places, different crop breeding enterprise, it is difficult to form effective operation mechanism, and greatly constrains the improvement of crop breeding management.

Another problem is how to generate value from crop breeding data. Before, a single source of information produce a single property data. With the development of intelligent sensor technology, it has become simple to collect various property crop breeding data, now, users concern was that whether these data at the right time, for the right purpose, and to provide right information?

Through the above introduction, in the future, in the process of development of crop breeding in China, there was an urgent need to regard and improve the following aspects: first, need to pay attention to the dynamic process that different physical equipment real-time acquisition and analysis data, and to effectively fuse the large data of crop breeding and establish related analytical model, multidimensional correlation, mining, prediction of multi-source data. Next, need to focus on the collaborative optimization of multi-issue, multi-link, and all-industrial chains about crop breeding, and translating data into information that could guide the production of crop breeding, and the use of information to produce optimized decisions and personalized services.

\section{Crop breeding architecture based on CPS}

\section{CPS summarize}

CPS (Cyber Physical System) was a kind of complex system that integrated computing, physical process and network communication, it has a clear framework and application flow sheet[14]. The meaning of CPS was many of physical entity equipment in the different location connected to the internet, so as to have these characteristics of "closely interconnected, intellisense and interactive synergy".

Main characteristic was shown as the following:

(1) Highly integrated about information and physical components;

(2)The physical components should have the ability about information processing and communication;

(3) It is a large-scale complex network system;

(4) Multiple dimensions complexity in time and space; 
(5)It could realize high dynamic organization and coordination of resources allocation.

(6) Self-learning, self- adaptive and dynamic autonomy, autonomous coordination.

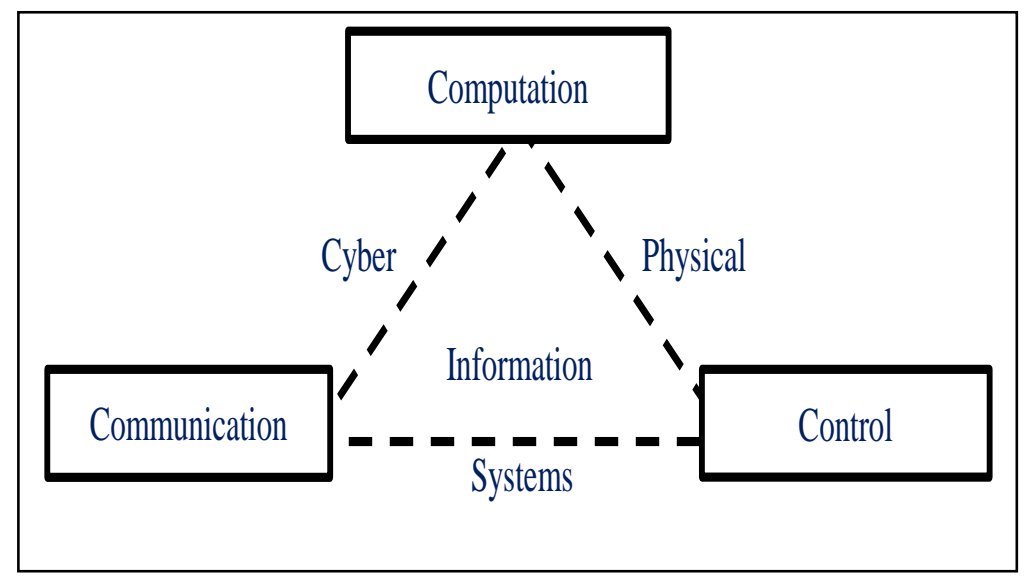

Fig.1 Abstraction of CPS

Figure 1 shows Abstraction of CPS(Cyber Physical System). The CPS was a multi-dimensional intelligence system, based on large data, network and huge amounts of calculation, through the core of intelligent perception, analysis, data mining, evaluation, prediction, optimization and coordination technology, realized deep combined with computing and communication and control(3C), to achieve the deep fusion of cyberspace and physical space.

\section{Crop breeding framework based on CPS}

In the process of crop breeding, crop show the various forms of complex traits, the whole process of crop breeding was a complex system with multiple elements, multidimensional properties, polymorphism, coexist about linear functions and nonlinear functions. Often, it was extremely rare that single causes lead to single consequences in the crop breeding process, and the actual cause and effect about crop breeding was almost necessarily a complex network structure. Traditional information, knowledge and related technologies were one dimension, and these were difficult to reveal the complex knowledge system of the whole process of crop breeding. At the same time, the environment of crop breeding has the typical characteristics of spatio-temporal geographical phenomena, multiple factors influence each other, contains rich information between complicated geographical relationship. Therefore, it was necessary that research the data of crop breeding based on multiple factor relation, and realize the coordination of all three about module group and the genetic background and regional environment.

The model architecture of crop breeding based on the CPS in this paper, to realize the goal was: based on various physical experimental instruments and equipment (field equipment, laboratory equipment, etc.), and a large number of software analysis tools distributed in different areas, these physical equipment and software analysis tools to be closely coupled through the CPS architecture, and to make intelligent decisions.

In this, crop breeding technology architecture based on CPS, it was including three layers: perceptual layer, network layer, cognitive layer. Figure 2 shows the Crop breeding system based on the CPS framework.

1 Perceptual layer

In perceptual layer, in order to achieve precision and efficient crop breeding, the first thing was how to use efficient and reliable ways to collect crop breeding data. In the perceptual layer, it included various kinds of sensors, test analysis and other physical equipment in the field and laboratory. Under the crop breeding environment, these data may come from different physical sources, including controllers, sensors, and so on. These data must be converted to meaningful information of crop breeding, for further evaluation and diagnosis. 


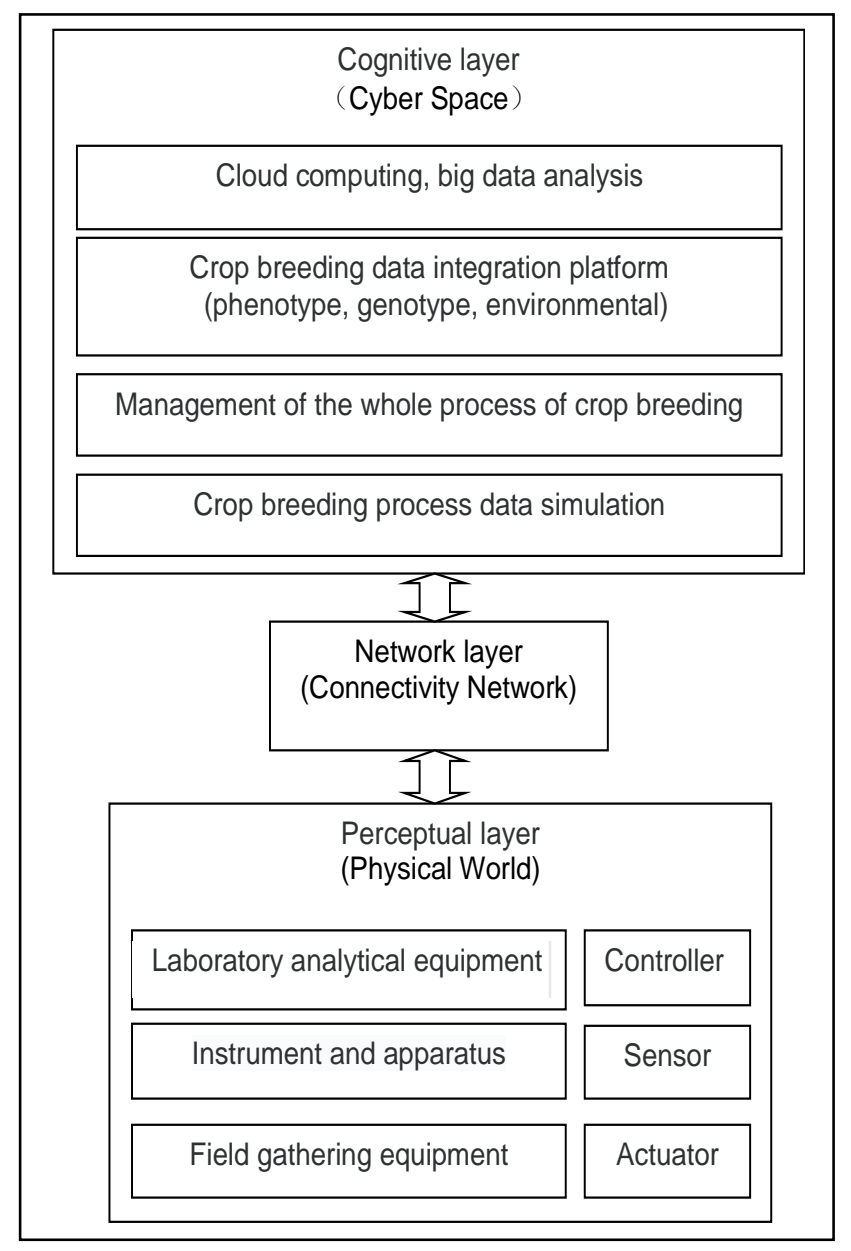

Fig.2 Crop breeding system based on the CPS framework

\section{Network layer}

In Network layer, we could harvest information from the relevant physical device, how to use it was the next challenge. If it could be able to comparison crop breeding data from other different physical device, users would be able to more deeply understand the various stages of crop breeding related the change of actual condition, and on this basis, further prediction possible development and change status in the process of crop breeding in the future. The network layer, through the content of the network management, and construction the effective transmission and the exchange of information of each physical device.

\section{Cognition layer}

In cognition layer, on the one hand, the physical layer at the front has the ability to respond to online real-time monitoring, not only could identify potential problems in different stages of crop breeding, but also had the ability and method to solve problems in real time. On the other hand, according to the evaluation and related adaptive learning, the system could make use of some specific prediction algorithm to predict the potential information, find solutions and make targeted decisions.

In this architecture, it was by no means a simple dissected physical device and information analysis system, that was precisely what the organic fusion of Physical World and Cyber Space, "you have me, and I in you". On the one hand, as a front-end physical device, they were not only the collection of data, but also the ability to analyze, process, feedback and control, could do some simple analysis. On the other hand, as the cognitive layer, its greatest feature was to implement the distributed management and coordinated decision of the analysis software and algorithmic model.

Finally, realized the ability of self-configuration and self-adjusting, self-optimization. From crop breeding technology based on the CPS, its core lies in the ability of data analysis to create new value, 
therefore, it also determines the CPS technology high versatility, applications could involve breeding workshop, field system, laboratory and other various fields.

\section{The advantages of CPS architecture in crop breeding applications}

For the many of crop breeding people, this paper built a multi-level integrated collaborative service system under the whole chain of crop breeding based on CPS, it could not only meet the demand of related crop breeding enterprise internal information, and also could achieve resource sharing, collaborative breeding and joint test between the alliance of crop breeding. Crop breeding model based on the CPS framework, it could effectively integrated all kinds of key technologies in the core application of crop breeding, such as intelligent data acquisition and integration of crop breeding technology, complex multidimensional numerical modeling technology, multi-level comprehensive analysis technology, multi-tasking multi-objective collaborative optimization techniques etc. For the whole process of crop breeding, building the integration innovation model of crop breeding of "intelligent equipment + comprehensive data analysis + intelligent information service", and then forming the ability to all-factor crop breeding data perceived ability, multi-dimensional data analysis and information service.

The specific performance is in the following aspects:

(1)The system could realize automatic control and intelligent information services between different regions and different equipment. It was able to control a wide variety of crop breeding hardware equipment that distributed different region and had different functions, so as to realize hardware equipment automatic perception, automatic discovery, automatic adjustment and intelligent control. Through the crop breeding system architecture based on CPS, it could realize that the information depth perception, intelligent optimization decision and precision control function etc. At the same time, it could effectively improve production efficiency, improve product quality, and reduce cost and consumption.

(2)The system could able to resolve the problem of integrity, timeliness and accuracy about phenotype data and genotype data type and environmental data in the whole process of crop breeding. Environment data, along with genotype data and phenotype data, form the basis of crop breeding large data. A lot of crop breeding data have certain spatial and temporal sequence characteristics. The each link of crop breeding existed many of data, data type was rich, but the data accuracy was not high, unable to support advanced analysis and intelligent optimization. The system could able to use advanced embedded system and sensor technology, enhance the ability to collect data from different time, different geographic regions, and different crop breeding stage, realize real-time acquisition and intelligent depth perception of large data for crop breeding. Figure 3 shows intelligent analysis and testing about big data of crop breeding.

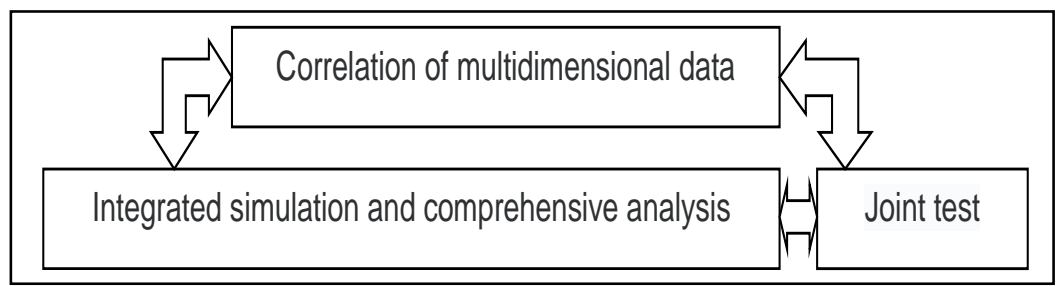

Fig.3 Intelligent analysis and testing about big data of crop breeding

(3)The system could improve on the ability of calculate the analysis capability and the application ability of crop breeding data. A lot of various features and functions of computational tools and analytical methods of crop breeding scattered in different breeding enterprise and relevant scientific research institutions, leading to large data analysis and practical application of crop breeding ability was too scattered, and could not deeper decision-making and optimizing these vast amounts of crop breeding data. Figure 4 shows feedback and control about Information in the whole process of crop breeding. The system could provide distributed crop breeding data analysis platform, through large data mining, cloud computing and other information technology, further enhance the level of 
calculation and analysis of crop breeding data, and more accurate analysis and master the knowledge of crop breeding, improve intelligent application level to the whole process of crop breeding, and thus for the majority of enterprises, scientific research units and users to provide more efficient service.

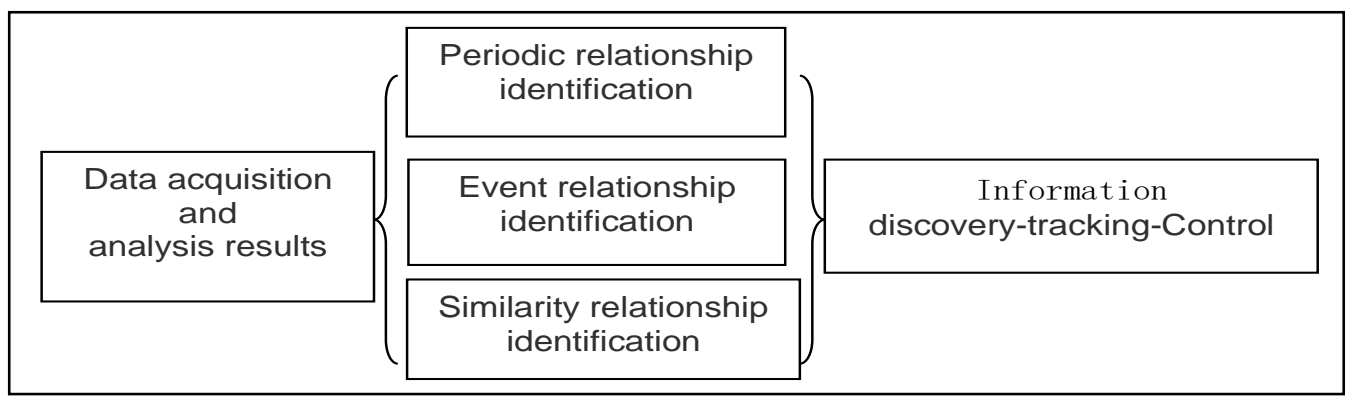

Fig.4 Feedback and control about Information in the whole process of crop breeding

The essence of crop breeding system architecture based on the CPS was to realize the "self-awareness, self-memory, self-cognitive, self-decision", physical substances (such as all kinds of sensor, controller, etc.) was the leading factor, information technology was the core, focus on building the knowledge platform and the intelligent application of control system, and finally realizes the intelligent system of crop breeding based on CPS.

\section{Conclusion}

Intelligent crop breeding was the only way to upgrade China's crop breeding enterprise, and it was also one of the main applications of big data and Internet of things in agriculture. Intelligent crop breeding not only involves computer and network technologies introduce the whole process of crop breeding, but also mean a full-scale revolution in research and production patterns about crop breeding. We should be aware that a lot of key technologies need our continuously research during realize the whole process of intelligent crop breeding in our country, on the one hand, the front end physical device needs to constantly update and perfect, on the other hand, tools, techniques and methods about building the CPS system need to improve. How to capture the phenotype data, genotype data, environment data, and space-time series big data and so on, on this basis, using big data analysis method to set up the relational model about data entity equipment and process of crop breeding, and by using information of the relational crop breeding model to management the whole process of crop breeding and creation services value, these would become the core of competitiveness in the future. To this end, we should have a good understanding of this.

Through the discussion above, with digital and standardization process of crop breeding, building modular and distributed technology process through the CPS platform, using the CPS service model to guide the crop breeding behavior of crop breeding enterprises, realize the shift from workshops breeding to commercial breeding, the shift from experience breeding to data breeding, the system would promote the upgrading of the commercial breeding technology system in low cost and high efficiency way. Using crop breeding technology architecture and application strategy based on CPS, realize the deep fusion of physical observation equipment and intelligent information processing, further enhance the competitiveness of crop breeding in our country and open up new market opportunities.

\section{Acknowledgements}

This research was supported by Research and application technology of commercialized breeding big data analysis by the Beijing municipal science and technology plan(D151100004215003).National 863 Programs Funded by Ministry of Science and Technology of China(2013AA102305). 


\section{References}

[1] Sun M, Goggi S A, Matson K, et al. Thin plate spline regression model used at early stages of soybean breeding to control field spatial variation[J]. Journal of Crop Improvement, 2015, 29(3):333-352.

[2] Khanal NP, Maharjan KL, Institutionalization of community seed production, Community Seed Production Sustainability in Rice-Wheat Farming. Springer, 2015: 163-172.

[3] Palmgren MG, Edenbrandt AK, Vedel SE, Are we ready for back-to-nature crop breeding? Trends in Plant Science,2015.20(3):155-164

[4] Connor D J, Wright G C, Dieters M J, et al. Development and application of speed breeding technologies in a commercial peanut breeding program[J]. Peanut Science, 2013, 40(2): 107-114.

[5] Gyibo S K, Naveen N D, Jerry S T. Effects of supply chain management practices on the performance of seed companies[J]. Asian Journal of Research in Business Economics and Management, 2015, 5(10): 39-49.

[6] Yang Xiao dong, Yu Xue ke, Huang Wei lin, Jiao Guang jing, The Status and Development Strategies of Commercial Breeding Crops in China Journal of Agriculture,2014,4(2):117-120

[7] GAI Junyi, LIU Kang, ZHAO Jinming, A Review on Advances in Science and Technology in Chinese Seed Industry, Scientia Agricultura Sinica,2015,48(17):3303-3315

[8] FAN Long jiang, WANG Wei di, WANG Bin, YE Chyu, Crop breeding-related data and application of big data technologies in crop breeding. Journal of Zhejiang University (Agric.\&Life Sci.), 2016,42(1):30-39

[9] Chen Wei, Guo Shupu. Current situation and existing problems of agricultural informatizationin China[C]. Transactions of the Chinese Society of Agricultural Engineering2013,22:196-204

[10]Cao Hong xin, ZhaoSuolao, GE Dao kuo, Discussion on development of agricultural models and digital agriculture[c],Jiangsu Journal of Agricultural Sciences,2012,5:1181-1188

[11]HOU Mandan, TONG Zhimin, ZHOU Aihong, LI Jingwen, A crop yield forecasting model based on discrete process neural networks and its application[c],Journal of Qiqihar University(Natural Science Edition),2013,4:44-52

[12]HUANG Qing,TANG Huaun,WU Wenbin,LI Dandan,LIUJia, Remote Sensing Based Dynamic Changes Analysis of Crop Distribution Pattern Taking Northeast China as an Example[c],Scientia Agricultura Sinica ,2013,13:101-119

[13]CAI Bin,MA Yufang,QIU Xiurong, Application Research on Internet of Things in Intelligent Agriculture[c],Modern Agricultural Science and Technology ,2013,14:23-31

[14]Tong Bo, Research on architecture of CPS system based on context-aware[D], Qingdao University of Science and Technology,2015.

[15]Liu Zhongqiang, Wang Kaiyi, Zhao Xiangyu, Li Minzan, Research on Information Model of Plant Breeding under Cloud Environment[J], Journal of Agricultural Mechanization Research ,2017,3:7-20

[16]Cheng Qihua,The Analysis and Design of Cyber-PhysicaI Systems Based on Cloud Computing[D],School of Computer Science and Technology Guangdong University of Technology,2015

[17]LI Ren-fa, YANG Fall, XIE Guoqi, Survey of modeling methods in cyber physical system[J],journal of communications,2016,37(5):165-175 
[18]LEE E A, Introduction to embedded systems a cyber-physical systems approach[M]. UC Berkeley, 2014: 79·200.

[19]ZHOU X S, YANG Y L, YANG G, Modeling methods for dynamic behaviors of cyber-physical system[J]. chinese Journal of Computers， 2014，37(3): l-12.

[20]YANG F, LI R, DUAN MQ, A modeling method research based on data in Cyber-physical system[J]. chinese Journal of Computers, 2015，38(136): l-15.

[21]YU Yang, JINYing, ZHANGJing, Modeling Method on Environmental Information in CPS, Journal of Jilin University ( ScienceEdition ),2015,53(2):280-286.

[22]Run Liu, Online Modeling and Verification of Dynamic Behavior Cyber-Physical Systems[D], Department of Computer Science and Technology Nanjing University,2016.

[23]SHI Chenbo, MIAO Quan, CHEN Qixin, Key technology and application of the energy Internet based on cyber physical systems[J],J Tsinghua Univ (Sci\&Technol), 2016, Vo1.56, No.9:930-937

[24]Li Chuqian, The analysis and design method of Cyber-Physical System based on big data[D],School of Computer Science and Technology Guangdong University of Technology,2015.

[25]Singh J, Hussain O, Chang E, Event handling for distributed real-time Cyber-Physical Systems[C]//EEE $15^{\text {th }}$ International Symposium on Object/Component/Service-Oriented Real-Time Distributed Computing, China, 2012: 23-30.

[26]Wang Yanan, WangFulin, ZhuHuixia, Design Research of the System about Remote Acquisition and Wireless Transmission for Field Information[C], ,Journal of Agricultural Mechanization Research,2013,3:88-95

[27]LIU Yangbin, YETao, ZHAOJintao, YangSaini, Hailfall risk assessment of crop damage by statistical modeling and random simulation: Case of HeBei[c],Journal of Beijing Normal University(Natural Science) ,2013,5:37-46 